\title{
The future of cardiac 123-I MIBG imaging
}

\author{
Arthur J. H. A. Scholte ${ }^{1}$
}

Published online: 16 September 2016

(C) Springer-Verlag Berlin Heidelberg 2016

The 123-I radiolabelled metaidobenzyguanidine (MIBG) is a norepinephrine analogue used for the functional assessment of the sympathetic nerve terminals of the heart. Planar scintigraphy parameters as the early (15 min post injection) and late ( $4 \mathrm{~h}$ post injection) heart to mediastinum $(\mathrm{H} / \mathrm{M})$ ratio as well as the washout ratio, and the defect score using single photon emission computed tomography (SPECT) are used to measure myocardial sympathetic function. The early $\mathrm{H} / \mathrm{M}$ ratio reflects the integrity of the presynaptic nerve terminals and the norepinephrine transport function of the synaptic cleft. The late $\mathrm{H} / \mathrm{M}$ ratio score combines information on neuronal function from uptake to release through the storage vesicle at the nerve terminals. Furthermore, myocardial 123-I MIBG washout is an index of the degree of sympathetic drive and increased sympathetic activity associated with high myocardial 123-I MIBG washout and low myocardial 123-I MIBG delayed uptake on scintigraphy and SPECT images. Reduction in myocardial uptake may reflect loss of nerve terminals (structural denervation) or down-regulation of the up-take 1 mechanism (dysfunctional). This cardiac 123-I MIBG imaging investigation has existed for more than 30 years, and, since 1980, hundreds manuscripts has been published with the main focus on heart failure. In this issue of the journal Gimelli et al. present the results of an interesting small study in which they assess the relationship between regional myocardial perfusion

This Editorial Commentary refers to the article http://dx.doi.org/10.1007/ s00259-016-3461-y.

Arthur J. H. A. Scholte

a.j.h.a.scholte@lumc.nl

1 Department of Cardiology, Leiden University Medical Center, Albinusdreef 2, P.O. box 9600, 2300 RC Leiden, The Netherlands and sympathetic innervation parameters at myocardial scintigraphy and intracavitary electrophysiological data in sixteen patients with ventricular arrhythmias (VA) submitted to invasive electrophysiological study and ablation procedure [1]. Although initial investigations have suggested the interplay between combined myocardial perfusion and sympathetic innervation abnormalities and inducibility of VA, a comprehensive assessment of the interaction among regional myocardial innervation/perfusion impairment and refined intra-cavitary left ventricular electrical parameters has not been performed before $[2,3]$. Myocardial fibrosis, surrounded by viable tissue and a trigger mechanism are the requirements for VA. Neuroregulation by both the sympathetic innervation as well as the parasympathetic innervation play probably, among other mechanisms, a central role in the development of VA. This concept is interesting, and since VA will be an increasing health care problem in patients with structural heart disease, there is a potential role for non-invasive nuclear imaging techniques to guide the ultimate ablation target in the ventricle. Moreover, three-dimensional 123-I MIBG cardiac innervation maps also may provide critical information about triggers/ substrate modifiers and improve understanding of VA and facilitate ablation [4]. So yes, beyond heart failure there are more potential clinical indications for the use of cardiac I 123I MIBG imaging [5].

In 2013 the FDA approved, after the results of the prospective ADMIRE-HF study, the use of 123-I MIBG in HF patients to measure the $\mathrm{H} / \mathrm{M}$ ratio to predict risk at 1 and 2- years [6]. However, since there are no studies that evaluated the impact of cardiac sympathetic innervation assessed by MIBG on treatment decisions for heart failure or that evaluated whether managing decisions that improve health outcomes are lacking, reimbursement by health insurance companies will remain. This may change with the ADMIRE-ICD study which possibly will demonstrate the efficacy of 123-I MIBG 
imaging for appropriately guiding the decision of ICD implantation in patients with NYHA class II and III heart failure with a LVEF between 30 and $35 \%$ (Clincaltrials.gov NCT02656329).

On the other hand, the nuclear cardiology guidelines of the Japanese Circulation Society recommend 123-I MIBG scintigraphy for the management of HF patients, particularly for the assessment of HF severity and prognosis [7]. Indeed, joint efforts for standardization of data acquisition and processing even as prospective randomized trials showing improvement in clinical outcome among patients who underwent cardiac I- 123 MIBG imaging are required and may help to improve the integration globally in practical guidelines and current clinical practice [8]. If the abovementioned obstacles are successfully tackled, other potential cardiac clinical indications, like, for instance, guidance of treatment of VA may follow. If not, cardiac 123-I MIBG imaging outside Japan will stay in a research setting and not be an essential management tool and probably finally disappear. In my opinion, this will be a great loss for the field of nuclear cardiology and should be prevented by joint efforts of ASNC, EANM, EACVI, and JSNC in cooperation with industries.

\section{Compliance with ethical standards}

Conflicts of interests The author declares that he has no conflicts of interests.

\section{References}

1. Gimelli AA, Menichetti F, Soldati E, et al. Relationships between cardiac innervation/perfusion imbalance and ventricular arrhythmias: impact on invasive electrophysiological parameters and ablation procedures. (1619-7089 (Electronic)).

2. Bax JJ, Kraft OF, Buxton AE, Fjeld JG, et al. 123 I-mIBG scintigraphy to predict inducibility of ventricular arrhythmias on cardiac electrophysiology testing: a prospective multicenter pilot study. (1942-0080 (Electronic)).

3. Zhou Y, Zhou WF, Folks RD, Manatunga D, et al. I-123 mIBG and Tc-99m myocardial SPECT imaging to predict inducibility of ventricular arrhythmia on electrophysiology testing: a retrospective analysis. (1532-6551 (Electronic)).

4. Klein T, Abdulghani M, Smith M, et al. Three-dimensional 123Imeta-iodobenzylguanidine cardiac innervation maps to assess substrate and successful ablation sites for ventricular tachycardia: feasibility study for a novel paradigm of innervation imaging. (19413084 (Electronic))

5. Dimitriu-Leen AC, Scholte AJ. Cardiac 123I-MIBG imaging beyound heart faiulure: potential clinical indications. Annals of Nuclear Cardiology. 2016;2(1):138-145.

6. Jacobson AF, Senior RF, Cerqueira MD, Wong N, et al. Myocardial iodine-123 meta-iodobenzylguanidine imaging and cardiac events in heart failure. Results of the prospective ADMIRE-HF (AdreView Myocardial Imaging for Risk Evaluation in Heart Failure) study. (1558-3597 (Electronic)).

7. Nakajima K, Nakata T. Cardiac 123I-MIBG Imaging for Clinical Decision Making: 22-Year Experience in Japan. (1535-5667 (Electronic)).

8. Verberne HJ. Assessment of cardiac sympathetic innervation with (123)I-mIBG SPECT comes to life: need for standardization! (2047-2412 (Electronic)). 